\title{
Transversity in the chiral quark-soliton model and single spin asymmetries*
}

\author{
A. V. Efremov** \\ ${ }^{1}$ Joint Institute for Nuclear Research, Dubna 141980, Russia
}

Key words QCD, partons, polarization, chiral model.

PACS 13.65.Ni, 13.60.Hb, 13.87.Fh, 13.88.+e

Dedicated to 60-th birthday of Prof. Klaus Goeke in collaboration with whom most described results were obtained.

A short review of single spin asymmetries in deep inelastic semi-inclusive processes, connected with prediction of chiral quark-soliton model for the nucleon transversity distributions, its possible theoretical understanding in the framework of QCD-induced approach and arising difficulties is given.

\section{Introduction}

It is well known that three most important (twist-2) elements of the parton density matrix in a nucleon are the non-polarized parton distributions functions (PDF) $f_{1}(x)$, the longitudinal spin distribution $g_{1}(x)$ and the transverse spin distribution (transversity) $h_{1}(x)$ [1]. The first two have been successfully measured experimentally in classical deep inelastic scattering (DIS) experiments but the measurement of the last one is especially difficult since it belongs to the class of the so-called chiral-odd structure functions and can not be seen there.

The non-polarized PDF's have been measured for decades and are rather well known in wide range of $x$ and $Q^{2}$. Their behavior in $Q^{2}$ is well described by the QCD evolution equation and serves as one of the main sources of $\alpha_{s}\left(Q^{2}\right)$ determination.

The longitudinal spin PDF's drew common attention during last decade in connection with the famous "Spin Crisis", i.e. astonishingly small portion of the proton spin carried by quarks (see [2] and references therein). The most popular explanation of this phenomenon is large contribution of the gluon spin $\Delta G(x)$. The direct check of this hypothesis is one of the main problems of running dedicated experiments like COMPASS at CERN and RHIC at BNL. Even now, however, there is some indication to a considerable value of $\Delta G(x)$ coming from the $Q^{2}$ evolution of the polarized PDF's [3] and from the first direct experimental probe of $\Delta G(x)$ by HERMES collaboration [4] with the result $\Delta G(x) / G(x)=0.41 \pm 0.18$ in the region $0.07<x<0.28$. The latter is in reasonable agreement with large $N_{c}$ limit prediction [5] $\Delta G(x) / G(x) \approx 1 / N_{c}$ for not very small $x$.

Another problem here is the sea quark spin asymmetry. It is usually assumed in fitting the experimental data that $\Delta \bar{u}=\Delta \bar{d}=\Delta \bar{s}$. This ad hoc assumption however contradicts with large $N_{c}$ limit prediction $\Delta \bar{u} \approx-\Delta \bar{d}[5]$. This was previously discovered in the instanton model [6] and

\footnotetext{
* The main results of this work was obtained in collaboration with K.Goeke and P.Schweitzer, Institut für Theoretische Physik II, Ruhr-Universität Bochum, Germany.

** E-mail: efremov@thsun1.jinr.ru
} 
supported by calculations in the chiral quark soliton model $(\chi \mathrm{QSM})[7,8]$. An indication to a nonzero value for $\Delta \bar{u}-\Delta \bar{d}$ was also observed in [9].

Concerning the transversity distribution it was completely unknown experimentally till recent time. The only information comes from the Soffer inequality [10] $\left|h_{1}(x)\right| \leq \frac{1}{2}\left[f_{1}(x)+g_{1}(x)\right]$ which follows from density matrix positivity. To access these chiral-odd structure functions one needs either to scatter two polarized protons and to measure the transversal spin correlation $A_{N N}$ in Drell-Yan process that is the problem for running RHIC and future PAX (GSI) experiments ${ }^{1}$ or to know the transverse polarization of a quark scattered from transversely polarized target. There are several ways to do this:

1. To measure the polarization of a self-analyzing hadron to which the quark fragments in a semi inclusive DIS (SIDIS), e.g. $\Lambda$-hyperon. The drawback of this method however is a rather low rate of quark fragmentation into $\Lambda$-particle $(\approx 2 \%)$ and especially that it is mostly sensitive to $s$-quark polarization. Also the polarization transfer from parton to $\Lambda$-hyperon is unknown.

2. To measure a transverse handedness in multi-particle parton fragmentation [12], i.e. the correlation of the parent quark spin 4 -vector $s_{\mu}$ and jet particle momenta $k_{i}^{\nu}, \epsilon_{\mu \nu \sigma \rho} s^{\mu} k_{1}^{\nu} k_{2}^{\sigma} k^{\rho}$ $\left(k=k_{1}+k_{2}+k_{3}+\cdots\right.$ is a jet 4 -momentum).

3. To use a new spin dependent T-odd parton fragmentation function $(\mathrm{PFF})[13,14,15]$ responsible for the left-right asymmetry in one particle fragmentation of transversely polarized quark relative to the quark momentum-spin plane. (The so-called "Collins asymmetry" [16].)

The last two methods are comparatively new and only in the last years some experimental indications to the transversal handedness [17] and to the T-odd PFF [18] have appeared.

Concerning the new PDF's and PFF's. Analogous of PDF's $f_{1}, g_{1}$ and $h_{1}$ are the PFF's $D_{1}, G_{1}$ and $H_{1}$, which describe the fragmentation of a non-polarized quark into a non-polarized hadron and a longitudinally or transversely polarized quark into a longitudinally or transversely polarized hadron, respectively. These PFF's are integrated over the transverse momentum $\boldsymbol{p}_{h \perp}$ of a hadron with respect to a quark. With $\boldsymbol{p}_{h \perp}$ taken into account, new PFF's arise. Using the Lorentz- and $\mathrm{P}$-invariance one can write in the leading twist approximation 8 independent spin structures. Most spectacularly it is seen in the helicity basis where one can build 8 twist- 2 combinations, linear in spin matrices of the quark and hadron $\boldsymbol{\sigma}, \boldsymbol{S}$ with momenta $\boldsymbol{k}^{\prime}, \boldsymbol{p}_{h}$.

These PFF can be used to extract the information on the proton transversity distribution from azimuthal asymmetries in SIDIS with hadron production (pions and kaons) on a polarized nucleon target

$$
l+\vec{N} \rightarrow l^{\prime}+h+X
$$

recently observed by HERMES [19]-[22] and CLAS [23, 24] collaborations.

In this short review I present the results of works [25]-[30] on the SIDIS asymmetries that are connected with the transversity distributions predicted in $\chi \mathrm{QSM}[31]$.

\section{SIDIS kinematics and azimuthal asymmetries}

In the framework of the parton model the squared matrix element modulus of the process (1) is represented by the diagram in Fig.1 and can be written as a sum of products of $x$-dependent quark distribution functions in a nucleon, $x=\frac{Q^{2}}{2 p \cdot q}$, with $q=l-l^{\prime}, Q^{2}=-q^{2}$, and $z$-dependent quark fragmentation functions of scattered quark into hadron $h, z=\frac{p \cdot p_{h}}{p \cdot q}$. The kinematics of the process (1) is presented in Fig.2.

\footnotetext{
${ }^{1}$ For predictions see [11] and references therein.
} 


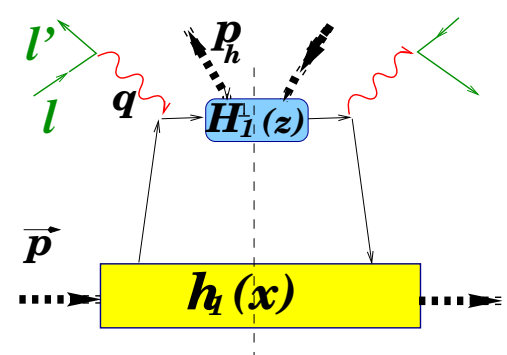

Fig. 1 The squared modulus of the matrix element of the process (1) in the parton model summed over states $X$. The $H_{1}^{\perp}(z)$ and $h_{1}(x)$ are examples of PFF and $\mathrm{PDF}$, respectively.

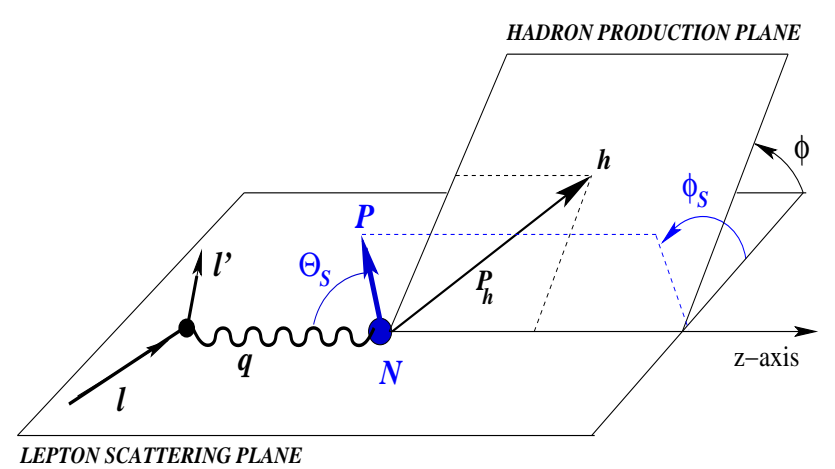

Fig. 2 Kinematics of the process (1).

The cross section of the semi-inclusive production of hadrons by polarized leptons on the polarized target is a linear function of the longitudinal lepton beam polarization, $P_{l}$, and the target polarization, $P$, with longitudinal, $P_{L}$, and transversal, $P_{T}$, components relative to a virtual photon momentum $\vec{q}$ in laboratory r.f.:

$$
d \sigma=d \sigma_{00}+P_{l} d \sigma_{L 0}+P_{L}\left(d \sigma_{0 L}+P_{l} d \sigma_{L L}\right)+\left|P_{T}\right|\left(d \sigma_{0 T}+P_{l} d \sigma_{L T}\right) .
$$

For the target polarization $P_{\|}$, longitudinal relative to the lepton beam, the transverse component is equal to $\left|P_{T}\right|=P_{\|} \sin \theta_{\gamma}$, where $\theta_{\gamma}$ is the angle of the virtual photon momentum $\vec{q}$ relative to the lepton beam,

$$
\sin \theta_{\gamma} \approx 2 \frac{M}{Q} x \sqrt{1-y},
$$

where $y=\frac{p \cdot q}{p \cdot l}$ and $M$ is the nucleon mass.

In the parton model each of the partial cross sections contributing to the Eq. (2) is characterized by the specific dependence on the azimuthal angle of an outgoing hadron, $\phi$, and on the azimuthal angle of transversal component of the target polarization vector ${ }^{2}, \phi_{S}$, relative to the lepton scattering plane (see Fig.2) times the definite product of PDF and PFF summed over quark and antiquark flavor times its charge squared. Namely, contributions to (2) for each quark and antiquark flavor up to the order $\mathcal{O}(M / Q))$ have the forms ${ }^{3}[13,14]$ :

$$
\begin{aligned}
d \sigma_{00} \propto & x f_{1}(x) D_{1}(z)+x h_{1}^{\perp}(x) H_{1}^{\perp}(z) \cos 2 \phi-\frac{M}{Q} x^{2} f^{\perp}(x) D_{1}(z) \cos \phi, \\
d \sigma_{L 0} \propto & \frac{M}{Q} x^{2} e(x) H_{1}^{\perp}(z) \sin \phi+\frac{M}{Q} x^{2} h_{1}^{\perp}(x) E(z) \sin \phi, \\
d \sigma_{0 L} \propto & x h_{1 L}^{\perp}(x) H_{1}^{\perp}(z) \sin 2 \phi+\frac{M}{Q} x^{2} h_{L}(x) H_{1}^{\perp}(z) \sin \phi, \\
d \sigma_{L L} \propto & x g_{1}(x) D_{1}(z)+\frac{M}{Q} x^{2} g_{L}^{\perp}(x) D_{1}(z) \cos \phi, \\
d \sigma_{0 T} \propto & x h_{1}(x) H_{1}^{\perp}(z) \sin \left(\phi+\phi_{S}\right)+x h_{1 T}^{\perp}(x) H_{1}^{\perp}(z) \sin \left(3 \phi-\phi_{S}\right) \\
& +x f_{1 T}^{\perp}(x) D_{1}(z) \sin \left(\phi-\phi_{S}\right), \\
d \sigma_{L T} \propto & x g_{1 T}(x) D_{1}(z) \cos \left(\phi-\phi_{S}\right),
\end{aligned}
$$

where

${ }^{2}$ For longitudinal target polarization $P_{\|}$the angle $\phi_{S}=0$ or $\pm \pi$

3 We use the notations of the work $[13,14,15]$. The letters $g_{1}\left(G_{1}\right), h_{1}\left(H_{1}\right), f_{1}\left(D_{1}\right)$ indicate twist-2 PDF (PFF) with longitudinally, transversally polarized or unpolarized partons, subscripts $L, T$ indicate the polarization of hadron and superscripts $\perp$ indicates a $p_{\perp}$-dependence. Note that very recently further structures have been introduced and discussed [32] that could contribute the longitudinally polarized cross sections. 
$f_{1}(x) \equiv q(x)$ is PDF of non-polarized quarks in a non-polarized target,

$g_{1}(x) \equiv \Delta q(x)$ is PDF of the longitudinally polarized quarks in the longitudinally polarized target,

$g_{1 T}(x)$ is the same as $g_{1}(x)$ but in the transversally polarized target,

$h_{1}(x)$ is PDF of the transversally polarized quark with polarization parallel to that one of a transversally polarized target (so-called transversity),

$h_{1 L, T}^{\perp}(x)$ is PDF of the transversally polarized quark with polarization perpendicular to the hadron polarization in the longitudinally or transversally polarized target,

$h_{1}^{\perp}(x)$ is PDF of the transversally polarized quark in the non-polarized target,

$f_{1 T}^{\perp}(x)$ is PDF responsible for a left-right asymmetry in the distribution of the non-polarized quarks in the transversally polarized target (so-called Sivers PDF [33]),

$D_{1}(z)$ is PFF of the non-polarized quark in the non-polarized or spinless produced hadron,

$H_{1}^{\perp}(z)$ is PFF responsible for a left-right asymmetry in the fragmentation of a transversally polarized quark into a non-polarized or spinless produced hadron (so-called Collins PFF [16]).

The others $\left(E, e, g_{L}^{\perp}, h_{L}, f^{\perp}\right)$ are the twist-3 functions entering with a factor $M / Q$. They have no definite probabilistic interpretation, but are connected (except for $e(x)$ and $E(z)$ ) to the above listed functions by the approximate integral relations of the Wandzura-Wilczek type. For example $[13,14]$

$$
\int d^{2} k_{\perp}\left(\frac{k_{\perp}^{2}}{2 M^{2}}\right) h_{1 L}^{\perp}\left(x, k_{\perp}\right) \equiv h_{1 L}^{\perp(1)}(x)=-(x / 2) h_{L}(x)=-x^{2} \int_{x}^{1} d \xi h_{1}(\xi) / \xi^{2} .
$$

Besides, in formula (4) each term should be multiplied by known kinematic factors depending on $y,\left\langle k_{\perp}\right\rangle$ and $\left\langle p_{h \perp}\right\rangle$ (assuming Gaussian distribution) which are omitted for simplicity.

The azimuthal asymmetries are defined as

$$
A_{B A}^{W\left(\phi_{,}\right)}(x, z, h)=\frac{\int \mathrm{d} y \mathrm{~d} \phi \mathrm{d} \phi_{S} W\left(\phi, \phi_{S}\right)\left(\frac{1}{P_{+}} \frac{\mathrm{d}^{4} \sigma_{D}^{+}}{\mathrm{d} x \mathrm{~d} y \mathrm{~d} z \mathrm{~d} \phi}-\frac{1}{P_{-}} \frac{\mathrm{d}^{4} \sigma_{D}^{+}}{\mathrm{d} x \mathrm{~d} y \mathrm{~d} z \mathrm{~d} \phi}\right)}{\frac{1}{2} \int \mathrm{d} y \mathrm{~d} \phi\left(\frac{\mathrm{d}^{4} \sigma_{D}^{+}}{\mathrm{d} x \mathrm{~d} y \mathrm{~d} z \mathrm{~d} \phi}+\frac{\mathrm{d}^{4} \sigma_{D}^{-}}{\mathrm{d} x \mathrm{~d} y \mathrm{~d} z \mathrm{~d} \phi}\right)},
$$

where $W\left(\phi, \phi_{S}\right)$ is an angular dependent weight from Eqs. (4) and $P_{ \pm}$denotes the target polarization modulus. The subscripts B and A are 0 , L or T for the unpolarized, longitudinally or transversally polarized beam or target (relative to the virtual photon direction).

It is clear that for the transversal target polarization one can separate all the components contained in (4) carrying out the Fourier-analysis with respect to the angles $\phi$ and $\phi_{S}$ plus change of the polarization sign (using anti-symmetrization and symmetrization). For example, for separation of the term with the pure transversity contribution $h_{1}(x) H_{1}^{\perp}(z)$ it is enough to calculate the average value $\left\langle\sin \left(\phi+\phi_{S}\right)\right\rangle$ and for separation of the Sivers function $f_{1 T}^{\perp}(x) D_{1}(z)$ it is enough to find the average value $\left\langle\sin \left(\phi-\phi_{S}\right)\right\rangle$.

For the longitudinal target polarization the total separation is impossible since $\phi_{S}= \pm \pi$ or 0 (see Fig.2) and several different mechanisms can produce the $\sin \phi$-asymmetry. However it is possible to single out the function $h_{1 L}^{\perp}(x)$, which is connected with transversity via Eq. (5), by measuring $\langle\sin (2 \phi)\rangle$. 


\section{Collins PFF}

As it is seen from (4) the Collins PFF that describes a left-right asymmetry in the fragmentation of a transversely polarized quark is especially interesting since it enters any term connected with the transversity. The corresponding term of fragmentation has the structure

$$
H_{1}^{\perp} \boldsymbol{\sigma}\left(\boldsymbol{k}^{\prime} \times \boldsymbol{p}_{h \perp}\right) / k^{\prime}\left\langle p_{h \perp}\right\rangle,
$$

where $H_{1}^{\perp}$ is a function of the longitudinal momentum fraction $z$ and hadron transverse momentum $p_{h \perp}$. The $\left\langle p_{h \perp}\right\rangle$ is the averaged transverse momentum of the final hadron ${ }^{4}$. Since the $H_{1}^{\perp}$ term is chiral-odd, it makes possible to measure the proton transversity distribution $h_{1}$ in semi-inclusive DIS from a transversely polarized target by measuring the left-right asymmetry of forward produced pions. The ratio $\frac{H_{1}^{\perp}}{D_{1}}$ serves as analyzing power of the Collins effect.

The problem is that, first, this function was completely unknown till recent time both theoretically and experimentally. Second, the function $H_{1}^{\perp}$ is the so-called T-odd fragmentation function: under the naive time reversal $\boldsymbol{p}_{h}, \boldsymbol{k}^{\prime}, \boldsymbol{S}$ and $\boldsymbol{\sigma}$ change sign, which demands a purely imaginary (or zero) $H_{1}^{\perp}$ in the contradiction with naive hermiticity. This, however, does not mean the break of T-invariance but rather the presence of an interference of different channels in forming the final state with different phase shifts, like in the case of single spin asymmetry phenomena ${ }^{5}$ [37]. A calculations of this function in simple perturbative chiral theory can be found in [38].

Meanwhile, the data collected by DELPHI (and other LEP experiments) give a possibility to measure $H_{1}^{\perp}$. The point is that despite the fact that the transverse polarization of quarks in $e^{+} e^{-} \rightarrow Z^{0} \rightarrow q \bar{q}$ is very small $\left(O\left(m_{q} / M_{Z}\right)\right)$, there is a non-trivial correlation $C_{T T}^{q \bar{q}}$ between transverse spins of a quark and an antiquark. In the Standard Model: $C_{T T}^{q \bar{q}}=\left(v_{q}^{2}-a_{q}^{2}\right) /\left(v_{q}^{2}+a_{q}^{2}\right)$, which are at $Z^{0}$ peak: $C_{T T}^{u, c} \approx-0.74$ and $C_{T T}^{d, s, b} \approx-0.35$. With the production cross section ratio $\sigma_{u} / \sigma_{d}=0.78$ this gives for the average over flavors value $\left\langle C_{T T}\right\rangle \approx-0.5$.

The transverse spin correlation results in a peculiar azimuthal angle dependence of produced hadrons, if the T-odd fragmentation function $H_{1}^{\perp}$ does exist [16, 39]. A simpler method has been proposed by Amsterdam group [15]. They predict a specific azimuthal behavior of a hadron in a jet about the axis in direction of another hadron in the opposite jet ${ }^{6}$

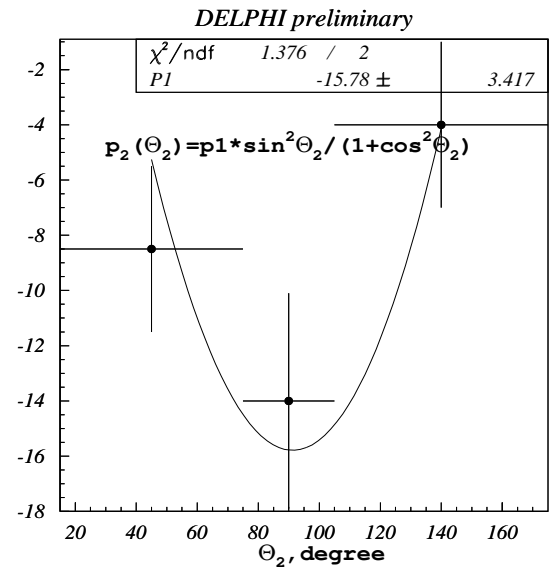

Fig. 3 The $\theta_{2}$-dependence of the value $P_{2}=\frac{6}{\pi}\left|\frac{\left\langle H_{1}^{\perp}\right\rangle}{\left\langle D_{1}\right\rangle}\right|^{2} \bar{C}_{T T} \frac{\sin ^{2} \theta_{2}}{1+\cos ^{2} \theta_{2}}$ (in ppm).

$$
\frac{\mathrm{d} \sigma}{\mathrm{d} \cos \theta_{2} \mathrm{~d} \phi_{1}} \propto\left(1+\cos ^{2} \theta_{2}\right) \cdot\left(1+\frac{6}{\pi}\left[\frac{H_{1}^{\perp q}}{D_{1}^{q}}\right]^{2} C_{T T}^{q \bar{q}} \frac{\sin ^{2} \theta_{2}}{1+\cos ^{2} \theta_{2}} \cos \left(2 \phi_{1}\right)\right),
$$

\footnotetext{
4 Notice different normalization factor compared with [15], $\left\langle p_{h \perp}\right\rangle$ instead of $M_{h}$.

5 In this aspect they are very different from the T-odd PDF's like $f_{1 T}^{\perp}$ or $h_{1}^{\perp}$ which can not exist since they are purely real. Interaction among initial hadrons which could brings an imaginary part breaks the factorization and the whole parton picture. Recently however it was stated [34] that effectively the necessary imaginary phase shift can appear due to propagation of the scattered parton in gluon field of the nucleon remnant. Since this phase shift depends on the subprocess the corresponding PDF is, generally speaking, not universal. In particular, it was shown that the Sivers PDF in one loop approximation, where its factorization seems proven $[35,36]$, should have opposite sign in Drell-Yan and SIDIS processes. Also this functions are suppressed in $\chi \mathrm{QSM}$ (see footnote 7).

6 The factorized Gaussian form of $p_{h \perp}$ dependence was assumed both for $H_{1}^{q \perp}$ and $D_{1}^{q}$ and integrated over $\left|p_{h \perp}\right|$.
} 
where $\theta_{2}$ is the polar angle of the electron beam relative to the second hadron momenta $\boldsymbol{p}_{2}$, and $\phi_{1}$ is the azimuthal angle of the first hadron counted off the $\left(\boldsymbol{p}_{2}, \boldsymbol{e}^{-}\right)$-plane. This asymmetry was probed [18] using the DELPHI data collection 91-95. For the leading charged particles (mostly pions) in each jet of two-jet events, summed over $z$ and averaged over quark flavors (assuming $H_{1}^{\perp}=\sum_{H} H_{1}^{\perp q / H}$ is flavor independent), the most reliable preliminary value of the analyzing power (obtained from the region $45^{\circ}<\theta_{2}<135^{\circ}$ with small acceptance corrections) is found to be $\left|\frac{\left\langle H_{1}^{\perp}\right\rangle}{\left\langle D_{1}\right\rangle}\right|=(6.3 \pm 2.0) \%$. However, the larger "optimistic" value

$$
\left|\frac{\left\langle H_{1}^{\perp}\right\rangle}{\left\langle D_{1}\right\rangle}\right|=(12.5 \pm 1.4) \%
$$

(obtained from the whole acceptable region $15^{\circ}<\theta_{2}<165^{\circ}$, see Fig. 3) is not excluded, with smaller statistical but presumably large systematic errors. This value, as it will be seen below, better fits the description of the azimuthal asymmetries in SIDIS.

\section{Chiral quark-soliton model prediction for $h_{1}^{a}(x)$}

In order to make quantitative estimates for asymmetries we will use for the transversity distribution function predictions from the chiral quark-soliton model ( $\chi$ QSM) [31]. This model was derived from the instanton model of the QCD vacuum [40] and describes numerous nucleonic properties without adjustable parameters to within $(10-30) \%$ accuracy [41]. The field theoretic nature of the model allows to consistently compute quark and antiquark distribution functions [7] which agree with parameterizations [42] to within the same accuracy. This gives us a certain confidence that the model also describes $h_{1}^{a}(x)$ with a similar accuracy.

In the $\chi$ QSM we observe the hierarchy $h_{1}^{u}(x) \gg$ $\left|h_{1}^{d}(x)\right| \gg\left|h_{1}^{\bar{u}}(x)\right|$, and an interesting "maximal sea quark flavour asymmetry" $h_{1}^{\bar{d}}(x) \approx-h_{1}^{\bar{u}}(x)>0$. In Fig. 4a we show the $\chi$ QSM prediction for $h_{1}^{a}(x)$ from Ref. [31] LO-evolved from the low scale of the model of about $\mu_{0}^{2}=(0.6 \mathrm{GeV})^{2}$ to the scale $Q^{2}=16 \mathrm{GeV}^{2}$. In order to gain some more intuition on the predictions we compare in Fig. $4 \mathrm{~b}$ the dominating distribution function $h_{1}^{u}(x)$ from the $\chi$ QSM to $f_{1}^{u}(x)$ and $g_{1}^{u}(x)$ from the parameterizations of Ref. [42]. It is remarkable that the Soffer inequality $\left|h_{1}^{u}(x)\right| \leq\left(f_{1}^{u}+g_{1}^{u}\right)(x) / 2$ is nearly saturated - in particular in the large- $x$ region. (The Soffer bound in Fig. 4b is constructed from $f_{1}^{u}(x)$ and $g_{1}^{u}(x)$ taken at $Q^{2}=16 \mathrm{GeV}^{2}$ from [42] in comparison with $h_{1}^{u}(x)$.)

For the unpolarized distribution function $f_{1}^{a}(x)$ we use the LO parameterization from Ref. [42].
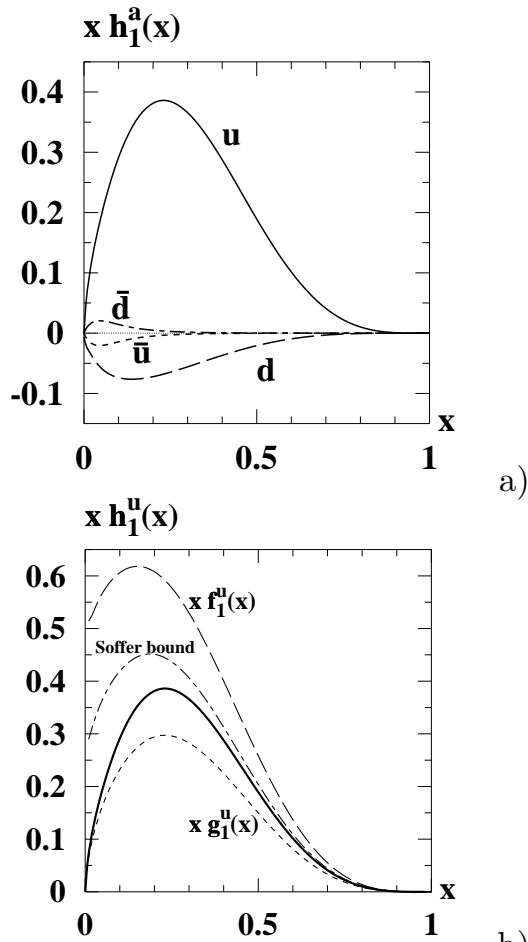

b)

Fig. 4 a) The transversity distribution function $h_{1}^{a}(x)$ vs. $x$ from the $\chi$ QSM [31]. b) Comparison of $h_{1}^{u}(x)$ from the $\chi$ QSM (solid) to $f_{1}^{u}(x)$ (dashed) and $g_{1}^{u}(x)$ (dotted) and the Soffer bound $\left(f_{1}^{u}+g_{1}^{u}\right)(x) / 2$ (dashed-dotted line) taken or constructed from the parameterizations of [42]. 


\section{Comparison with longitudinally polarized data}

In the HERMES experiment the $x$ - and $z$-dependence of asymmetries (6) for pions and kaons with $W=\sin \phi$ or $\sin 2 \phi$ from longitudinally (relative to the non-polarized lepton beam) polarized proton and deuterium target were measured. As it is seen from (4) only the second term of $d \sigma_{0 L}$ and the first (Collins) and the third (Sivers) ones ${ }^{7}$ of $d \sigma_{0 T}$ with factor (3) do contribute the numerator of (6) for $W=\sin \phi$ with negative sign $\left(\phi_{S}=\pi\right)$ and only the third one of the $d \sigma_{0 L}$ contributes for $W=\sin 2 \phi$. This gives for asymmetries

$$
\begin{aligned}
& A_{0 L, P \text { or } D}^{\sin \phi}(x, z, h)=P_{L}(x) \frac{\sum_{a} e_{a}^{2} x h_{L}^{a / P \circ \mathrm{or} D}(x) H_{1}^{\perp a}(z)}{\sum_{a}^{h} e_{a}^{2} f_{1}^{a / P \circ \mathrm{or} D}(x) D_{1}^{a}(z)}-P_{T}(x) \frac{\sum_{a} e_{a}^{2} h_{1}^{a / P_{\mathrm{or}} D}(x) H_{1}^{\perp a}(z)}{\sum_{a}^{h} e_{a}^{2} f_{1}^{a / P \circ \mathrm{or} D}(x) D_{1}^{a}(z)} \\
& A_{0 L, P \text { or } D}^{\sin 2 \phi}(x, z, h)=P_{1}(x) \frac{\sum_{a} e_{a}^{2} h_{1 L}^{\perp(1), a / P \circ \mathrm{or} D}(x) H_{1}^{\perp a}(z)}{\sum_{a}^{h} e_{a}^{2} f_{1}^{a / P \circ \mathrm{or} D}(x) D_{1}^{a}(z)}
\end{aligned}
$$

where $P_{L}(x)$ and $P_{T}(x)$ are known factors of order $\mathcal{O}(M / Q)$ and $P_{1}$ of order $\mathcal{O}(1)$ depending on average transverse momenta of partons inside hadron and hadrons inside parton. The functions $h_{L}^{a}(x)$ and $h_{1 L}^{\perp(1), a}(x)$ are expressed through transversity $h_{1}^{a}(x)$ by the relation (5).

For $H_{1}^{\perp a}$ and $D_{1}^{a}$ a strong suppression of the unfavoured with respect to the favoured fragmentation has been assumed. From charge conjugation and isospin symmetry one has then

$$
H_{1}^{\perp \text { fav }} \equiv H_{1}^{\perp u / \pi^{+}}=H_{1}^{\perp \bar{d} / \pi^{+}}=H_{1}^{\perp d / \pi^{-}}=2 H_{1}^{\perp u / \pi^{0}} \cdots \gg H_{1}^{\perp d / \pi^{+}}=H_{1}^{\perp \bar{u} / \pi^{+}} \cdots \equiv H_{1}^{\perp \mathrm{unf}} .
$$

So, using the DELPHI result ${ }^{8}$ Eq.(8), $\chi$ QSM for $h_{1}^{a}(x)$, the relation (5) for the $h_{L}$ and the parameterization from Ref. [42] for $f_{1}^{a}(x)$, both LO-evolved to the average scale $Q_{\mathrm{av}}^{2}=4 \mathrm{GeV}^{2}$ characteristic for HERMES we obtain for the proton target Fig. 5
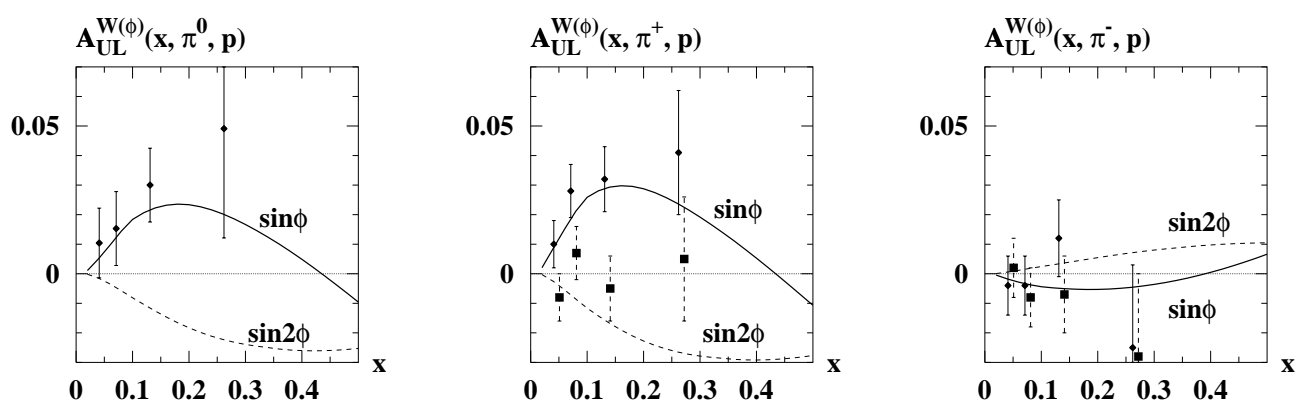

Fig. 5 Azimuthal asymmetries $A_{0 L}^{W(\phi)}(x, \pi)$ weighted by $W(\phi)=\sin \phi$ (solid line) and $\sin 2 \phi$ (dashed line) for the production of $\pi^{0}, \pi^{+}$and $\pi^{-}$as function of $x$. The experimental data are from Refs. [19, 20]. Rhombus (squares) denote data for $A_{0 L}^{\sin \phi}\left(A_{0 L}^{\sin 2 \phi}\right)$.

7 Actually, our approach would imply the vanishing of the Sivers effect. This is in agreement with the $\chi$ QSM. However, this cannot be taken literally as a prediction for the following reason. The $\chi$ QSM was derived from the instanton vacuum model as the leading order in terms of the instanton packing fraction $\frac{\rho}{R} \sim \frac{1}{3}(\rho$ and $R$ are respectively the average size and separation of instantons in Euclidean space time). In this order the T-odd PDF's like $f_{1 T}^{\perp}$ and $h_{1}^{\perp}$ vanish [43]. In higher orders the T-odd PDF's can be well non-zero and all one can conclude at this stage is that the T-odd PDF's are suppressed with respect to the T-even. However, considering that $H_{1}^{\perp}(z)$ is much smaller than $D_{1}(z)$, cf. Eq. (8), it is questionable whether this suppression could be sufficient such that in physical cross sections the Collins effect $\propto h_{1}^{a}(x) H_{1}^{\perp}(z)$ is dominant over the Sivers effect $\propto f_{1 T}^{\perp}(x) D_{1}(z)$. (For an estimation of this suppression see $[28,44]$.)

8 We assume a weak scale dependence of the analyzing power (8). 


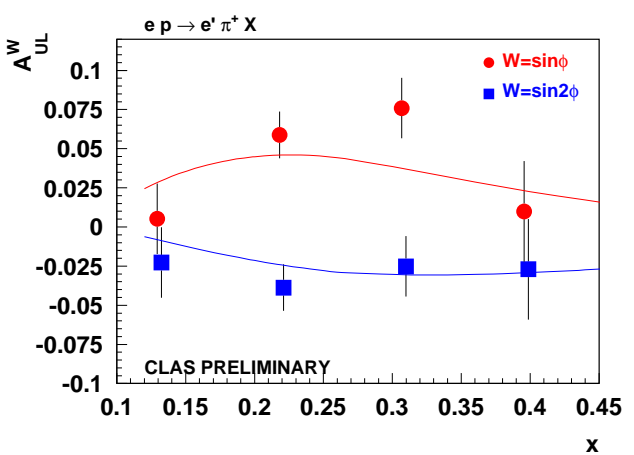

Fig. 6 Our predictions (solid curves) for azimuthal asymmetries $A_{0 L, D}^{\sin 2 \phi}$ and $A_{0 L, D}^{\sin \phi}$ vs. $x$ in comparison with CLAS data [23].

\section{$\mathbf{H}(\mathbf{z}) / \mathbf{D}(\mathbf{z})$}

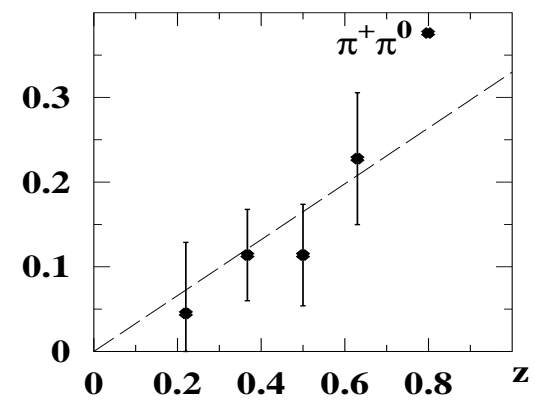

Fig. $7 H_{1}^{\perp} / D_{1}$ vs. $z$, as extracted from HERMES data $[19,20]$ for $\pi^{+}$and $\pi^{0}$ production combined.
Fig. 6 shows also our predictions for $A_{0 L, P}^{\sin \phi}(x, \pi)$ and $A_{0 L, P}^{\sin 2 \phi}(x, \pi)$ in comparison with CLAS data [23] for a $5.7 \mathrm{GeV}$ beam.

We conclude that the azimuthal asymmetries obtained with the $\chi \mathrm{QSM}$ prediction for $h_{1}^{a}(x)$ [31] combined with the "optimistic" DELPHI result (8) for the analyzing power are consistent with experiment with no fit parameter.

It is interesting to note that the negative sign of the transversal contribution in (9) leads to a change of sign of the $A_{0 L}^{\sin \phi}$ asymmetries for $x>0.4$. This is due to a harder behaviour $h_{1}(x)$ with respect to $h_{L}(x)$ followed from Eq. 5 . It should be noted that the prediction of $A_{0 L}^{\sin \phi}(x, \pi)=0$ at $x \simeq(0.4-0.5)$ is sensitive to the approximation of favoured flavour fragmentation (11). In principle one could conclude from data, how well this approximation works. However, the upper $x$-cut is $x<0.4$ in the HERMES experiment [19, 20].

Now let us reverse the logic, and using z-dependence of the HERMES results for the $\pi^{0}$ and $\pi^{+}$azimuthal asymmetries try to estimate $H_{1}^{\perp}(z) / D_{1}(z)$. For that we use the $\chi \mathrm{QSM}$ prediction for $h_{1}^{a}(x)$ that will introduce a model dependence of order $(10-20) \%$. The combined result is shown in Fig. 7. The data can be described by a linear fit

$$
H_{1}^{\perp}(z)=(0.33 \pm 0.06) z D_{1}(z)
$$

with average $\left\langle H_{1}^{\perp}\right\rangle /\left\langle D_{1}\right\rangle=(13.8 \pm 2.8) \%$ which is in good agreement with DELPHI result Eq.(8). The errors are the statistical errors of the HERMES data. It is interesting to note that numerically the behavior (12) is close to those calculated from chirally invariant Manohar-Georgi model [38], $H_{1}^{\perp}(z) \approx 0.63 z^{2} D_{1}(z)$.

Believing that such acceptable description of the proton data is not occasional we made the predictions [27] for $A_{0 L}$ asymmetries for pions and kaons at longitudinally polarized deuteron target which were being measured at that time by the HERMES collaboration.

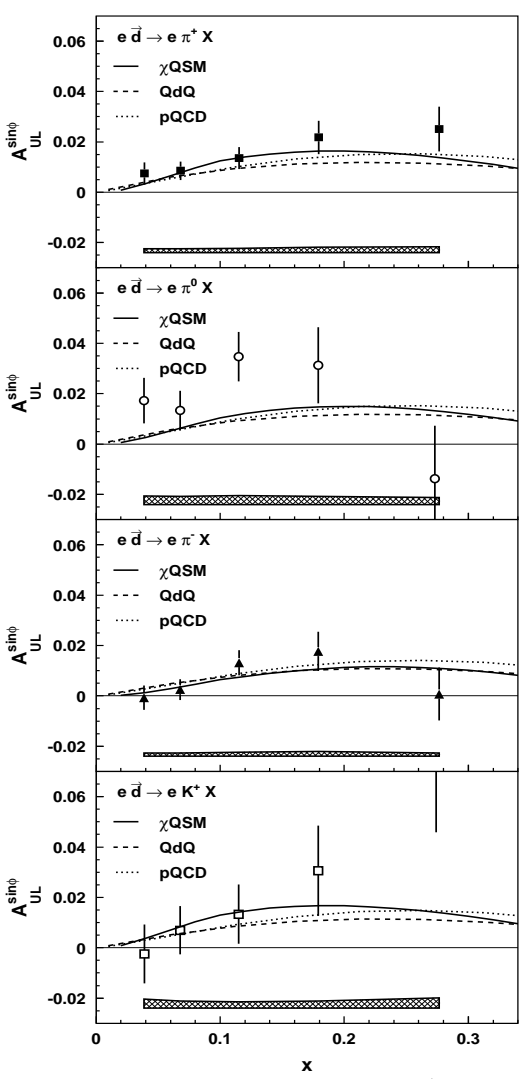

Fig. 8 Our predictions (solid curves) for $A_{0 L, D}^{\sin \phi}(x, h)$ vs. $x$ from a longitudinally polarized deuteron target in comparison with HERMES data [21]. 
The main question was, however, how large is the analyzing power for kaons? We know that the unpolarized kaon fragmentation function $D_{1}^{K}(z)$ is roughly five times smaller than the unpolarized pion one. Is also $H_{1}^{\perp} K(z)$ five times smaller than $H_{1}^{\perp} \pi(z)$ ? The reason is that in chiral limit $D_{1}^{\pi}=D_{1}^{K}$ and $H_{1}^{\perp \pi}=H_{1}^{\perp K}$. The naive expectation is that the 'way off chiral limit to real world' proceeds analogously as for spin-dependent quantities, $H_{1}^{\perp}$, as for spin-independent one, $D_{1}$. If we assume this, i.e. if

$$
\frac{\left\langle H_{1}^{\perp K}\right\rangle}{\left\langle D_{1}^{K}\right\rangle} \simeq \frac{\left\langle H_{1}^{\perp \pi}\right\rangle}{\left\langle D_{1}^{\pi}\right\rangle}
$$

holds, we obtain - with the central value of $\left\langle H_{1}^{\perp}\right\rangle /\left\langle D_{1}\right\rangle$ in Eq.(8) - azimuthal asymmetries for $K^{+}$ and $K^{0}$ as large as for pions. The results of our predictions [27] (solid curves) in comparison with the published HERMES data [21] are presented at Fig. 8. Again no fit parameters were used in distinction with other models at Fig. 8. The asymmetries for $\bar{K}^{0}$ and $K^{-}$are close to zero in our approach.

\section{Extraction of $\mathbf{e}(x)$ from $\mathbf{A}_{\mathbf{L} 0}$}

Very recently the $\sin \phi$ asymmetry of $\pi^{+}$produced by scattering of polarized electrons off unpolarized protons was published by CLAS collaboration [24] and preliminary data were reported by HERMES collaboration [22]. This asymmetry is interesting since it allows to access the unknown twist-3 structure functions $e^{a}(x)$ (see $d \sigma_{L 0}$ in (4)) that are connected with the nucleon $\sigma$ term:

$$
\int_{0}^{1} \mathrm{~d} x \sum_{a} e^{a}(x)=\frac{2 \sigma}{m_{u}+m_{d}} \approx 10 .
$$

The asymmetry is given by ${ }^{9}$

$$
A_{L 0}^{\sin \phi}(x) \propto \frac{M}{Q} \frac{\sum_{a} e_{a}^{2} e^{a}(x)\left\langle H_{1}^{\perp a / \pi}\right\rangle}{\sum_{a} e_{a}^{2} f_{1}^{a}(x)\left\langle D_{1}^{a / \pi}\right\rangle} .
$$

Disregarding unfavored fragmentation and using the Collins analysing power extracted from HERMES in (12) that yields for $z$-cuts of CLAS $\left\langle H_{1}^{\perp \pi}\right\rangle /\left\langle D_{1}^{\pi}\right\rangle=0.20 \pm 0.04$, we can extract

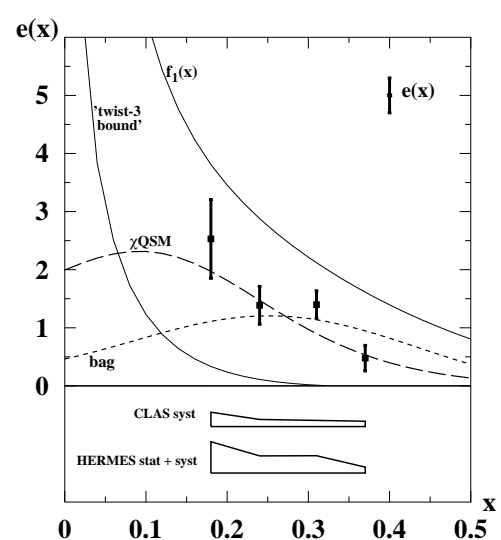

Fig. 9 The flavour combination $e(x)=\left(e^{u}+\frac{1}{4} e^{\bar{d}}\right)(x)$, with error bars due to statistical error of CLAS data, vs. $x$ at $\left\langle Q^{2}\right\rangle=1.5 \mathrm{GeV}^{2}$. For comparison the twist-3 bound, $f_{1}^{u}(x)$, bag and $\chi$ QSM models predictions are shown. [30] $e^{u}(x)+\frac{1}{4} e^{\bar{d}}(x)$. The result is presented in Fig. 9. For comparison the twist-3 lower bound ${ }^{10}$, $e^{a}(x) \geq 2\left|g_{T}^{a}(x)\right|-h_{L}^{a}(x)$ Ref. [10], and the unpolarized distribution function $f_{1}^{u}(x)$ are plotted. The prediction of $\chi$ QSM [45] and bag model [46] are shown also. One can guess that the large number in the sum rule Eq.(14) might be due to, by all means, a $\delta$-function at $x=0$. (For review and references see [47].)

\footnotetext{
9 The term $\propto \sum_{a} e_{a}^{2} h_{1}^{\perp a}(x)\left\langle E^{a / \pi}\right\rangle$ in numerator was disregarded since $h_{1}^{\perp a}$ are suppressed in $\chi$ QSM (see footnote 7.)

10 Let us stress that strictly speaking this inequality could be justified only if the "twist-2 Soffer inequality" $2\left|h_{1}^{a}(x)\right| \leq\left(f_{1}^{a}+g_{1}^{a}\right)(x)$ of Ref. [10] were saturated [47]. In the following we will refer to this relation as "twist-3 lower bound" keeping in mind that it does not need to hold in general. In Ref. [48] a bound based on the positivity of the hadronic tensor and the Callan-Gross relation (and formulated in terms of structure functions) was discussed.
} 

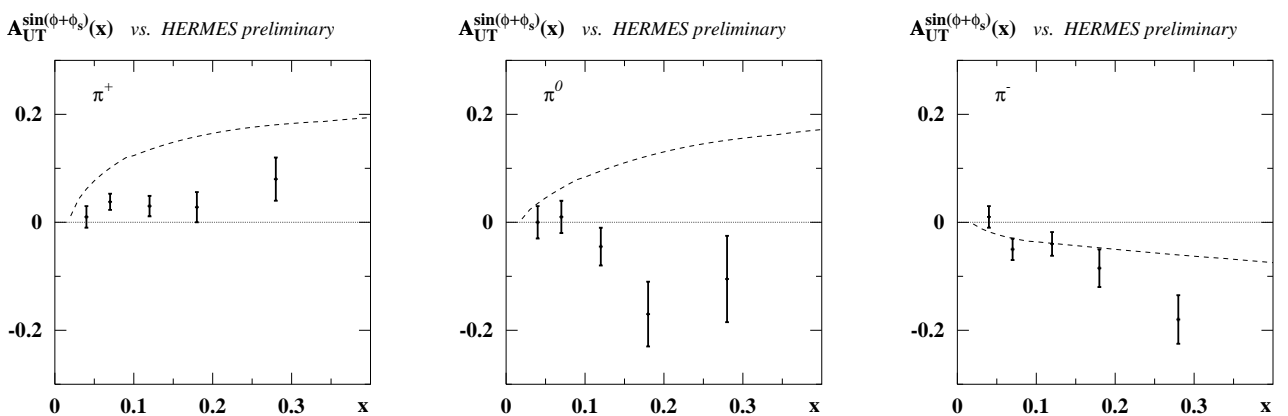

Fig. 10 The Collins effect transverse target SSA $A_{0 T}^{\sin \left(\phi+\phi_{s}\right)}$ in the production of $\pi^{+}, \pi^{0}$ and $\pi^{-}$from a proton target. Preliminary data are from [49], theoretical curves from [29].

\section{SIDIS with transverse target polarization}

In the HERMES and COMPASS experiments the cross sections $\sigma_{N}^{\uparrow \downarrow}$ for the process $l N^{\uparrow \downarrow} \rightarrow l^{\prime} h X$ is measured, where $N^{\uparrow \downarrow}$ denotes the transversely relative to the beam polarized target (see Fig. 2).

The component of the target polarization vector which is transverse relative to the hard photon is characterized by the angle $\Theta_{S}$, see Fig. 2, given by

$$
\sin \Theta_{S}=\cos \theta_{\gamma} \sqrt{1+\tan ^{2} \theta_{\gamma} \sin ^{2} \phi_{S^{\prime}}} \approx \cos \theta_{\gamma} \approx 1
$$

where $\phi_{S}^{\prime}$ is the azimuthal angle of the target polarization direction about the lepton beam direction relative to the scattering plane. As it is seen from (4) only the first (Collins) term of $d \sigma_{0 T}$ with factor (16) does contribute to the numerator of (6) for $W=\sin \left(\phi+\phi_{S}\right)$. This gives for Collins asymmetry

$$
A_{0 T}^{\sin \left(\phi+\phi_{s}\right)}(x, z, h)=B_{T}(x) \frac{\sum_{a} e_{a}^{2} x h_{1}^{a}(x) H_{1}^{\perp a}(z)}{\sum_{b} e_{b}^{2} x f_{1}^{b}(x) D_{1}^{b}(z)},
$$

where $B_{T}(x)$ are known factors order of $\mathcal{O}(1)$ depending on average transverse momenta.

Based on our understanding of the longitudinally polarized target asymmetry, predictions for the Collins effect for transversally polarized target asymmetry $A_{0 T}^{\sin \left(\phi+\phi_{s}\right)}$ were made ${ }^{11}[29]$ (see Fig. 10). Of course, since the theoretical description of the power suppressed ("twist-3") longitudinal asymmetry is involved and we made simplifications, which are difficult to control, one cannot expect that we accurately predict the overall magnitude of the effect. However, one could have a certain confidence that at least the sign and the shape of $A_{0 T}^{\sin \left(\phi+\phi_{s}\right)}(x)$ is described satisfyingly since it is dictated by the model prediction for $h_{1}^{a}(x)$ [31] and the approximation of favoured flavour fragmentation only. As can be seen in Fig. 10 our results [29] do not even describe the sign for $\pi^{0}$ of the preliminary HERMES data [49]! Why not?

Apparently some assumption(s) made must be incorrect. The first suspicion is favoured fragmentation approximation (11). First of all, pay attention to the negative and large $A_{0 T}^{\sin \left(\phi+\phi_{s}\right)}\left(\pi^{0}\right)$. With the unfavored fragmentation taken into account one has from charge conjugation and isospin invariance (11)

$$
H_{1}^{\perp a / \pi^{0}}=\frac{1}{2}\left(H_{1}^{\perp a / \pi^{+}}+H_{1}^{\perp a / \pi^{-}}\right)=\frac{1}{2}\left(H_{1}^{\perp \text { fav }}+H_{1}^{\perp \text { unf }}\right) .
$$

11 Asymmetries of similar magnitude as for HERMES are also predicted [29] for the running COMPASS experiment with transversally and longitudinally polarized targets. 
Then

$$
\underbrace{A_{0 T}^{\sin \left(\phi+\phi_{s}\right)}\left(\pi^{0}\right)}_{<0 \text { in experiment }} \propto \underbrace{\sum_{a} e_{a}^{2} h_{1}^{a}(x)}_{>0 \text { in models }}\left\langle H_{1}^{\perp \text { fav }}+H_{1}^{\perp \text { unf }}\right\rangle \Longrightarrow\left\langle H_{1}^{\perp \text { fav }}+H_{1}^{\perp \text { unf }}\right\rangle<0 .
$$

In order to explain the asymmetry for charged pions the option $H_{1}^{\perp \text { fav }}<0$ can be ruled out, unless $\left(4 h_{1}^{u}+h_{1}^{\bar{d}}\right)<\left(h_{1}^{d}+4 h_{1}^{\bar{u}}\right)$ which would contradict any model. Then, with option $H_{1}^{\perp \text { fav }}>0$, we can draw two interesting conclusions from the observation in Eq. (19). Firstly, $H_{1}^{\perp \text { unf }}$ should have opposite sign with respect to $H_{1}^{\perp \text { fav }}$. This could have a natural explanation in string models, in particular for the HERMES kinematics [49] with low particle multiplicity jets. Secondly, the absolute value of $H_{1}^{\perp \text { unf }}$ has to be larger than the absolute value of $H_{1}^{\perp \text { fav }}$ which, if confirmed, will be more difficult to understand.

Concerning the large value of $A_{0 T}^{\sin \left(\phi+\phi_{s}\right)}\left(\pi^{0}\right)$ notice that it is approximately of the same order as $A_{0 T}^{\sin \left(\phi+\phi_{s}\right)}\left(\pi^{-}\right)$. Meanwhile from the factorization of $x$ and $z$ dependence of polarized and unpolarized SIDIS cross sections and from relation (18) one can write for any spin asymmetry

$$
A\left(\pi^{0}\right)=\frac{\sigma\left(\pi^{+}\right)}{\sigma\left(\pi^{+}\right)+\sigma\left(\pi^{-}\right)} A\left(\pi^{+}\right)+\frac{\sigma\left(\pi^{-}\right)}{\sigma\left(\pi^{+}\right)+\sigma\left(\pi^{-}\right)} A\left(\pi^{-}\right)=A\left(\pi^{-}\right)+a\left[A\left(\pi^{+}\right)-A\left(\pi^{-}\right)\right],
$$

where $\sigma(\pi)$ is unpolarized SIDIS cross section. If $A\left(\pi^{0}\right) \approx A\left(\pi^{-}\right)$then $a=\frac{\sigma\left(\pi^{+}\right)}{\sigma\left(\pi^{+}\right)+\sigma\left(\pi^{-}\right)} \approx 0$ that is nonsense! This leads to conclusion that the factorization of $x$ and $z$ dependence for the transversally polarized SIDIS cross sections is under suspicion and should be carefully checked. Regrettably the statistical errors are rather large, especially for $A_{0 T}\left(\pi^{0}\right)$. Probably due to this reason the final publication [50] do not contain $A_{0 T}\left(\pi^{0}\right)$. But even without $\pi^{0}$ the question stays why $A_{0 T}\left(\pi^{+}\right)$is so small and $\left|A_{0 T}\left(\pi^{-}\right)\right|$is so large since unfavored $\left\langle H_{1}^{\perp \text { unf }}\right\rangle$ gives opposite sign contributions to $\pi^{+}$and $\pi^{-}$asymmetries the latter about order of magnitude larger.

The present situation seems paradoxical. We have a reasonable understanding of $A_{0 L}$ asymmetries, but know that it possibly is based on an incomplete theoretical description of the process with the Sivers effect and other contributions omitted. We probably have a more reliable description of the Collins $A_{0 T}$ asymmetry, but cannot understand the preliminary data.

However, one should keep in mind the preliminary stage of the data [49], which does not allow yet to draw more definite conclusions. Further data from HERMES as well as COMPASS, CLAS, HALL-A and HALL-B experiments will contribute considerably to resolve the present puzzles and pave the way towards a qualitative understanding of the numerous new distribution and fragmentation functions.

\section{Conclusion}

Processes with polarized particles have always been among the most difficult and complicated themes both for experimentalists and theorists.

First, working with polarized targets, experimentalists have to "battle with" thermal chaos which tends to break the polarized order. For this one needs liquid helium temperatures. More difficulties, like depolarizing resonances, are encountered in accelerating polarized particles and in controlling a polarized beam. Second, spin effects are very perfidious: as a rule, they are strongest in kinematical regions where the process itself is the least probable.

As for the theory, I can hardly recall a case when its first prediction was correct! As a rule, it was wrong and forced theorists to think more fundamentally to repair the theory. This resulted in a deeper understanding of particle interaction mechanics. Nevertheless many puzzles such as "Why are hyperons produced so strongly polarized?" or "What is the structure of the nucleon spin?" stay yet unsolved during decades. Will single spin asymmetries become one more such problem? The future will show. 
Acknowledgements The author is partially supported by grants RFBR 03-02-16816 and DFG-RFBR 03-02-04022.

\section{References}

[1] J.Ralston and D.E.Soper, Nucl.Phys B152 (1979) 109. J.L.Cortes, B.Pire, and J.P.Ralston, Z. Phys.C55 (1992) 409.

R.L.Jaffe and X.Ji, Phys. Rev. Lett. 67 (1991) 552; Nucl. Phys B375 (1992) 527.

[2] M.Anselmino, A.Efremov and E.Leader, Phys. Rep. 261 (1995) 1 [hep-ph/9501369].

[3] E.Leader, A.V.Sidorov and D.B.Stamenov, Phys. Lett. B462 (1999) 189 [hep-ph/9905512].

[4] A.Airapetian et al. [HERMES Collaboration], Phys. Rev. Lett. 84 (2000) 2584.

[5] A.V.Efremov, K.Goeke and P.V.Pobylitsa, Phys. Lett. B488 (2000) 182 [hep-ph/0004196].

[6] A.E.Dorokhov and N.I.Kochelev, Phys. Lett. B304 (1993) 167; A.E.Dorokhov, N.I.Kochelev and Yu.A.Zubov, Int. J. Mod. Phys. A8 (1993) 603.

[7] D. I. Diakonov, V. Yu. Petrov, P. V. Pobylitsa, M. V. Polyakov and C. Weiss, Nucl. Phys. B 480 (1996) 341 [arXiv:hep-ph/9606314]; Phys. Rev. D 56 (1997) 4069.

M. Wakamatsu and T. Kubota, Phys. Rev. D 60 (1999) 034020 [arXiv:hep-ph/9809443].

P. V. Pobylitsa, M. V. Polyakov, K. Goeke, T. Watabe and C. Weiss, Phys. Rev. D 59 (1999) 034024 [arXiv:hep-ph/9804436].

K. Goeke, P. V. Pobylitsa, M. V. Polyakov, P. Schweitzer and D. Urbano, Acta Phys. Polon. B 32, 1201 (2001) [arXiv:hep-ph/0001272].

[8] B.Dressler, K.Goeke, M.V.Polyakov and C.Weiss, Eur. Phys. J. C14 (2000) 147 [hep-ph/9909541].

[9] T.Morii and T.Yamanashi, Phys. Rev. D61 (2000) 057501.

[10] J. Soffer, Phys. Rev. Lett. 74, 1292 (1995).

[11] A. V. Efremov, K. Goeke and P. Schweitzer, Eur. Phys. J. C 35 (2004) 207 [arXiv:hep-ph/0403124]. M. Anselmino, M. Boglione, U. D'Alesio, E. Leader and F. Murgia, arXiv:hep-ph/0407100.

[12] A.Efremov, L.Mankiewicz and N.Törnqvist, Phys. Lett. B284 (1992) 394.

[13] P. J. Mulders and R. D. Tangerman, Nucl. Phys. B 461 (1996) 197 [Erratum-ibid. B 484 (1997) 538] [arXiv:hep-ph/9510301].

D.Boer and R.Tangerman, Phys. Lett. B381 (1996) 305.

[14] D. Boer and P. J. Mulders, Phys. Rev. D 57 (1998) 5780 [arXiv:hep-ph/9711485].

[15] D.Boer, R.Jakob and P.J.Mulders, Phys. Lett. B424 (1998) 143.

[16] J.Collins, Nucl. Phys. B396 (1993) 161.

X.Artru and J.C.Collins, Z. Phys. C69 (1996) 277.

[17] A.V.Efremov, Y.I.Ivanshin, L.G.Tkatchev and R.Y.Zulkarneev: Phys. Atom. Nucl. 63 (2000) 445 [nucl-th/9901005]. A.V.Efremov et al., Czech. J. Phys. 49 (1999) 75, Suppl. S2 [hep-ph/9901216].

[18] A.V.Efremov, O.G.Smirnova and L.G.Tkatchev, Nucl. Phys. (Proc. Suppl.) 74 (1999) 49 and 79 (1999) 554 [hep-ph/9812522].

[19] A. Airapetian et al. [HERMES Collaboration], Phys. Rev. Lett. 84 (2000) 4047 [arXiv:hepex/9910062].

[20] A. Airapetian et al. [HERMES Collaboration], Phys. Rev. D 64 (2001) 097101 [arXiv:hepex/0104005].

[21] A. Airapetian et al. [HERMES Collaboration], Phys. Lett. B 562 (2003) 182 [arXiv:hep-ex/0212039].

[22] E. Avetisyan, A. Rostomyan and A. Ivanilov [By HERMES Collaboration], [arXiv:hep-ex/0408002].

[23] H. Avakian and L. Elouadrhiri [for the CLAS Collaboration], "Single Spin asymmetries at CLAS," Proc. of $X$ Workshop on High Energy Spin Physics (NATO ARW DUBNA-SPIN-03), Dubna, Sept. 16-20, 2003, JINR E1,2-2004-80, pp. 239-248.

H. Avakian, "Spin and azimuthal asymmetries in DIS," Prepared for 10th Workshop on High-Energy Spin Physics: NATO Advanced Research Workshop, Nor Amberd, Armenia, 30 Jun - 3 Jul 2002

"Spin-azimuthal asymmetries in semiinclusive DIS," DESY-HERMES-00-17 Prepared for 8th International Workshop on Deep Inelastic Scattering and QCD (DIS 2000), Liverpool, England, 25-30 Apr 2000

[24] H. Avakian et al. [CLAS Collaboration], Phys. Rev. D 69 (2004) 112004 [arXiv:hep-ex/0301005].

[25] A. V. Efremov, K. Goeke, M. V. Polyakov and D. Urbano, Phys. Lett. B 478 (2000) 94 [arXiv:hep$\mathrm{ph} / 0001119]$. 
[26] A. V. Efremov, K. Goeke and P. Schweitzer, Phys. Lett. B 522 (2001) 37 [Erratum-ibid. B 544 (2002) 389] [arXiv:hep-ph/0108213].

[27] A. V. Efremov, K. Goeke and P. Schweitzer, Eur. Phys. J. C 24 (2002) 407 [arXiv:hep-ph/0112166]; Nucl. Phys. A 711 (2002) 84; Acta Phys. Polon. B 33 (2002) 3755 [arXiv:hep-ph/0206267].

[28] A. V. Efremov, K. Goeke and P. Schweitzer, Phys. Lett. B 568 (2003) 63 [arXiv:hep-ph/0303062].

[29] A. V. Efremov, K. Goeke and P. Schweitzer, Eur.Phys.J. C32 (2004) 337 [arXiv:hep-ph/0309209].

[30] A. V. Efremov, K. Goeke and P. Schweitzer, Phys. Rev. D 67 (2003) 114014 [arXiv:hep-ph/0208124].

[31] P. V. Pobylitsa and M. V. Polyakov, Phys. Lett. B 389 (1996) 350 [arXiv:hep-ph/9608434].

P. Schweitzer, D. Urbano, M. V. Polyakov, C. Weiss, P. V. Pobylitsa and K. Goeke, Phys. Rev. D 64 (2001) 034013 [arXiv:hep-ph/0101300]. AIP Conf. Proc. 549 (2002) 659.

[32] A. Afanasev and C. E. Carlson, arXiv:hep-ph/0308163.

F. Yuan, Phys. Lett. B 589 (2004) 28 [arXiv:hep-ph/0310279].

A. Metz and M. Schlegel, arXiv:hep-ph/0403182.

A. Bacchetta, P. J. Mulders and F. Pijlman, Phys. Lett. B 595, 309 (2004) [arXiv:hep-ph/0405154].

[33] D. W. Sivers, Phys. Rev. D 41 (1990) 83.

D. W. Sivers, Phys. Rev. D 43 (1991) 261.

[34] D. Boer, P. J. Mulders and O. V. Teryaev, Phys. Rev. D 57, 3057 (1998) [arXiv:hep-ph/9710223].

S. J. Brodsky, D. S. Hwang and I. Schmidt, Phys. Lett. B 530, 99 (2002).

J. C. Collins, Phys. Lett. B 536, 43 (2002).

A. V. Belitsky, X. Ji and F. Yuan, Nucl. Phys. B 656, 165 (2003).

[35] X. D. Ji, J. P. Ma and F. Yuan, arXiv:hep-ph/0404183, arXiv:hep-ph/0405085.

[36] J. C. Collins and A. Metz, arXiv:hep-ph/0408249.

[37] S.Gasiorowich, Elementary particle physics, Wiley, New-York, 1966, p. 515.

[38] A. Bacchetta, R. Kundu, A. Metz and P. J. Mulders, Acta Phys. Polon. B 33 (2002) 3761 [arXiv:hepph/0206309], Phys. Rev. D 65 (2002) 094021 [arXiv:hep-ph/0201091].

[39] J.Collins et al., Nucl. Phys. B420 (1994) 565.

[40] For reviews see: D. I. Diakonov, Prog. Part. Nucl. Phys. 51 (2003) 173 [arXiv:hep-ph/0212026]. D. I. Diakonov and V. Yu. Petrov, [arXiv:hep-ph/0009006].

[41] C. V. Christov et al., Prog. Part. Nucl. Phys. 37 (1996) 91 [arXiv:hep-ph/9604441].

[42] M. Glück, E. Reya and A. Vogt, Z. Phys. C 67 (1995) 433; Eur. Phys. J. C 5 (1998) 461. M. Glück, E. Reya, M. Stratmann and W. Vogelsang, Phys. Rev. D 53 (1996) 4775; Phys. Rev. D 63 (2001) 094005 [arXiv:hep-ph/0011215].

[43] P. V. Pobylitsa, arXiv:hep-ph/0212027.

[44] P. Schweitzer and A. Bacchetta, Nucl. Phys. A 732 (2004) 106 [arXiv:hep-ph/0310318].

[45] P. Schweitzer, Phys. Rev. D 67 (2003) 114010 [arXiv:hep-ph/0303011].

[46] A. I. Signal, Nucl. Phys. B 497, 415 (1997).

[47] A. V. Efremov and P. Schweitzer, JHEP 0308 (2003) 006 [arXiv:hep-ph/0212044].

[48] W. Lu and J. J. Yang, Z. Phys. C 73, 689 (1997) [arXiv:hep-ph/9601255].

[49] U. Elschenbroich, G. Schnell and R. Seidl [HERMES Collaboration], [arXiv:hep-ex/0405017]. R. Seidl [HERMES], Proc. of DIS'2004, 13-18 April, Štrbské Pleso, Slovakia. N. C. Makins [HERMES], talk at "Transversity Workshop", 6-7 Oct. 2003, Athens, Greece.

[50] A. Airapetian et al. [HERMES Collaboration], arXiv:hep-ex/0408013. 